\title{
BACKGROUND MODELLING USING A Q-TREE BASED FOREGROUND SEGMENTATION
}

\author{
SHAHIDHA BANU $\mathrm{S}^{*}$ AND MAHESWARI $\mathrm{N}^{\dagger}$
}

\begin{abstract}
Background modelling is an empirical part in the procedure of foreground mining of idle and moving objects. The foreground object detection has become a challenging phenomenon due to intermittent objects, intensity variation, image artefact and dynamic background in the video analysis and video surveillance applications. In the video surveillances application, a large amount of data is getting processed by everyday basis. Thus it needs an efficient background modelling technique which could process those larger sets of data which promotes effective foreground detection. In this paper, we presented a renewed background modelling method for foreground segmentation. The main objective of the work is to perform the foreground extraction only in the intended region of interest using proposed Q-Tree algorithm. At most all the present techniques consider their updates to the pixels of the entire frame which may result in inefficient foreground detection with a quick update to slow moving objects; The proposed method contract these defect by extracting the foreground object by controlling the region of interest (the region only where the background subtraction is to be performed) and thereby reducing the false positive and false negative. The extensive experimental results and the evaluation parameters of the proposed approach with the state of art method were compared against the most recent background subtraction approaches. Moreover, we use challenge change detection dataset and the efficiency of our method is analyzed in different environmental conditions (indoor, outdoor) from the CDnet2014 dataset and additional real time videos. The experimental results were satisfactorily verified the strengths and weakness of proposed method against the existing state-of-the-art background modelling methods.
\end{abstract}

Key words: Background modelling, dynamic background, foreground detection, background subtraction, Quad Tree segmentation

AMS subject classifications. $68 \mathrm{U} 10,94 \mathrm{~A} 08$

1. Introduction. The noteworthy increase in the number of cameras being used for the purpose of video analysis and surveillance is due to low cost of higher resolution cameras. However, some issues always hold back users related to the surveillance of the captured videos, for example the cost will be more as we maintain the recorded data a long duration. Thus it is required for a motion detection algorithm which forms basic working of background subtraction, which can further build a background model to which the current frame is compared.

The background subtraction algorithm is therefore required to discriminate foreground from background. The static part of the scene here is background and the moving objects are the foreground. While a static background model is enough to analyses short videos in a controlled indoor environment, it is insufficient for most practical cases; Therefore, a more refined model is required to handle dynamic background, background with shadow and illumination change. Moreover, the motion detection is on the preliminary step in any of the application related to surveillance. For example, finding the region where moving object is detected might be made available for the further analysis or processing of unattended objects, ghost removal, human monitoring, traffic control, etc. Thus, background subtraction (BS) is the normal approach to detect motion in videos. The procedure is termed as foreground segmentation which involves frame comparison, update to background model and foreground segmentation. This procedure produces a binary mask.

Background modeling $[18,19]$, is a process of considering the background under different condition. A good algorithm should correctly detect the moving object, and at the same time remove the shadow. Besides, it is also important that it must exactly extract the foreground having same color as the background. A "universal" background subtraction algorithm has to handle the following critical situations:

\footnotetext{
*Research Scholar, SCSE, VIT University - Chennai Campus, Chennai-600127, India (ssbanuphd@gmail.com)

${ }^{\dagger}$ Professor, SCSE, VIT University - Chennai Campus, Chennai-600127, India.
} 
- Noise in the image due to sudden and gradual lightning variation

- Detecting slow moving object as well intermittent objects where the non-static object stays for a period of time as the static object.

- Movement of objects in the background as well multiple foreground objects.

- Handling shadow region.

It is complicated to achieve these goals with a simple background subtraction, by design, that promotes at the pixel level for improved productivity and it is not easy to analyze significant change patterns to produce better results.

The BS algorithm performs the task in two stages: background modelling and foreground extraction. The modeling is all about the measured design of the background demonstration and a technique engaged to accommodate the background changes at different timing instant. The object detection refers to the method engaged for pixel classification; generally, it is a threshold process where the present value $\mathrm{x}$ of the active pixel is compared to the same pixel in the $\mathrm{B}(\mathrm{x})$ background model. Conferring to the calculated difference in pixel value, $\mathrm{x}$ may be classified as foreground when the calculated difference is greater than threshold and when it is relatively smaller the pixel $\mathrm{x}$ is considered to be background. Furthermore, this procedure indirectly involves a mechanism using feedback among the modeling and the foreground detection modules. It is also a critical phase in reducing the misclassifications and increasing the accuracy in foreground segmentation. This problem invades an open choice between various methods for updating the background pixels.

In this paper, we present an adaptive region based background modeling method for the efficient foreground segmentation. The proposed method detects the primary region into which background subtraction to be performed. Region adjustments are regulated by monitoring: 1) moving objects in the background; 2) similarity between observed foreground and background models; 3) instable region based on regular fluctuations; and 4) the transmission of changing brightness or so called illumination changes. These four regulations are used as algorithm framing guide for an efficient foreground segmentation technique. The rest of the paper is organized as follows: In Section II, we comprehensively review the literature of background modeling techniques. This work gives an idea about the other frameworks developed for the purpose. Some of the methods are implemented and results are taken for comparison with our routine. Section III labels our method and specifies the routines used in our major research work: the background model initialization process, finding the region of interest and the update policy. Section IV deliberates the metric parameters used, and the experimental results along with comparisons and execution performance.. We show that performing background subtraction in the specified region alone in its simplified form is equivalent the other techniques that are more sophisticated. The conclusion is given in Section $\mathrm{V}$.

2. Literature Review. Ample count of background modeling methods have been offered over the past decades to identify or to fragment the foreground objects in a video stream. They commonly follow the general procedure of considering the initaial frame or the mean of first few frames as background model, and then it finds the pixel based difference between the the current frame with the background model to sense the foreground objects. Various proposed modeling methods either parametric or Non-parametric were categorized into pixel-based, region or block based, and hybrid. One of the popular parametric methods which is also pixel-based is the Gaussian model. Wren et al. [1], which is considered to be first pixel based background modelling method.

However, a simple Gaussian function is not suitable to detect slow moving objects and frames with dynamic background due to lack of update mechanism to the background model [2]. Movements of tree leaves and water waves are considered to be dynamic background [3], and Stauffer and Grimson [4, 5] recommended a model that works on every pixel of the frame called the Gaussian mixture model (GMM). It is the procedure which works with mixture of K-mean Gaussians functions. As an improvement to above method, EM-based algorithm offered where it introduces a way to initialize the factor through online in the modelling of background, which is left to be time consuming. Adaptive Gaussian was proposed by Zivkovic, [30, 6] who anticipate an update to the parameters and D.S.Lee [7] has proposed a mechanism to improve the combining rate without diturbing the gaussian [5] stability as an adaptive learning rate. . Chien et al. [49] throws a threshold based factor for the object detection. Here, they consider the camera noise and set to zero mean in gaussian distribution since it is the only factor that affects the threshold. 
Shimada et al. [8] introduces a varying component to control the GMM in order to increase the accuracy and to reduce the computational time. Also, Oliver et al. [10] introduced a background modelling method based on Bayesian method which uses the previous information of data and keeps gathering proof from the dataConsidering the Non-Parametric category in background modeling methods, Maddalena et al. [18] proposed a nonparametric algorithm based artificial neural networks with self-organization (SOBS). Kim et al. [19, 20] proposed a method of codebook which performs background modelling that initializes codeword and stores the statitics of codeword used in the codebook. Barnich et al. [11, 12] projected a pixel-based algorithm from the category nonparametric named Vibe. It detects the moving object as foreground using a the pattern of sampling intensity and random selection strategy. Wang et al.[42] suggested a technique (SACON) using manipulative sample consensus based on every pixel of the statistical model. The model detects the foreground object by exploiting both color and motion information. The superior performance to many other state-of-theart methods is considered to be ViBe and is considered to be the exact method that detects the background changes in every frames [13]. The further study about the Vibe method was conceded by Droogenbroeck and Paquot [14] and they promote the performance enhancement by enhancing the ViBe with some considered additional constraints. Another such pixel based non parametric method proposed by Hofmann et al. [15] is pixel based adaptive segmenter (PBAS) method. The method keeps track of history of recent time observed pixel values and these are applied as background model to segment the foreground object. Although, the result of above discussed state-of-art methods projects well the outlines of foreground objects,i.e. the shape of the object and Also, they are affected by various factors like noise, dynamic background and lighting changes ,etc without any difficulty.

As an another approach to Pixel-based background modelling there exist theRegion-based methods.It uses the inter-pixel relations to enhance its efficiency and segments the images into regions and performs foreground objects identification from the segmented image regions. Kernel Estimation Density(KDE) projected by Elgammal et al. [9, 28] offered a novel method by building a nonparametric background model. Seki et al. [50] applied a different technique of background modelling by applying image variation co-occurrence in the defined image regions. A heuristic algorithm distinguishes the foreground object from the background based on block matching and similar method was proposed by Russell et al. [51] to . In which they compared fixed size of background regions with the input frames. In order to provide solution to the dynamic background modeling challenges in outdoor settings, Eng et al. [52] offered a random regular arrangements and image regions with pre filters, by using color space to detect foregrounds in the ClELab. Furthermore the method is featured by color and texture based analysis which receives a attractive attention among region based methods. An another background modelling method proposed by Heikkila et al. [53] engaged a technique called local binary pattern (LBP), which involves the discriminative texture feature of the image for modeling the background.In the above model, LBP histogram is built for partially overlapping regions and the same will be compared against the LBP histogram of every incoming frames regions. Liu et al. [54] proposed a method to resolve the challenge of illumination change. It is also an efficient background modeling method for foreground segmentation based on binary descriptor and proven to adapt under continuously varying illumination condition. In addition, another method based on binary descriptors was proposed by Huang et al. [55] as a sample based background modelling a replacement to parametric methods. In divergence to pixel-based foreground segmentation methods,these region-based segmentation methods can obtain only coarse shapes of foreground objects but can reduce the misclassification to a greater extent, thus reducing the false correlations .

3. Proposed Architecture for Q-tree based Intrusion Detection System. Here we have proposed an architecture for an Intrusion detection and prevention system. The Fig. 4.1 portrays the flow in the architecture. The flow includes the data acquisition from the camera attached in the surveillance area is then forwarded to the intrusion detection module which incorporate the proposed Q-Tree based foreground segmentation. Once the intrusion is detected in the surveillance area the intimation will be given to the provided webserver which further gets forwarded to the user by the webserver.

The data acquisition model collects the video data from the camera in the area under surveillance region and is given to the IDM(Intrusion detection model) which in turn incorporates our proposed approach using the concept of "Q-tree Image segmentation(QIS) ". In first subsection, we presented preprocessing technique, second subsection explains the Q-Tree Decomposition, third subsection focuses on the routine to find Region 


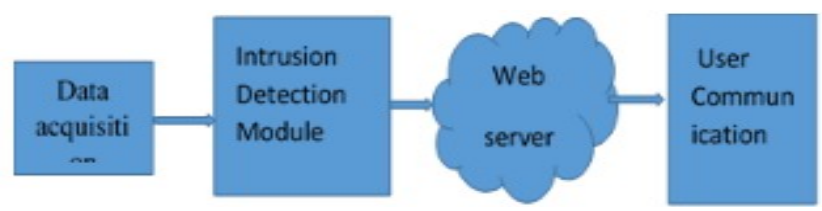

FIG. 3.1. General Architecture of the intrusion detection system.

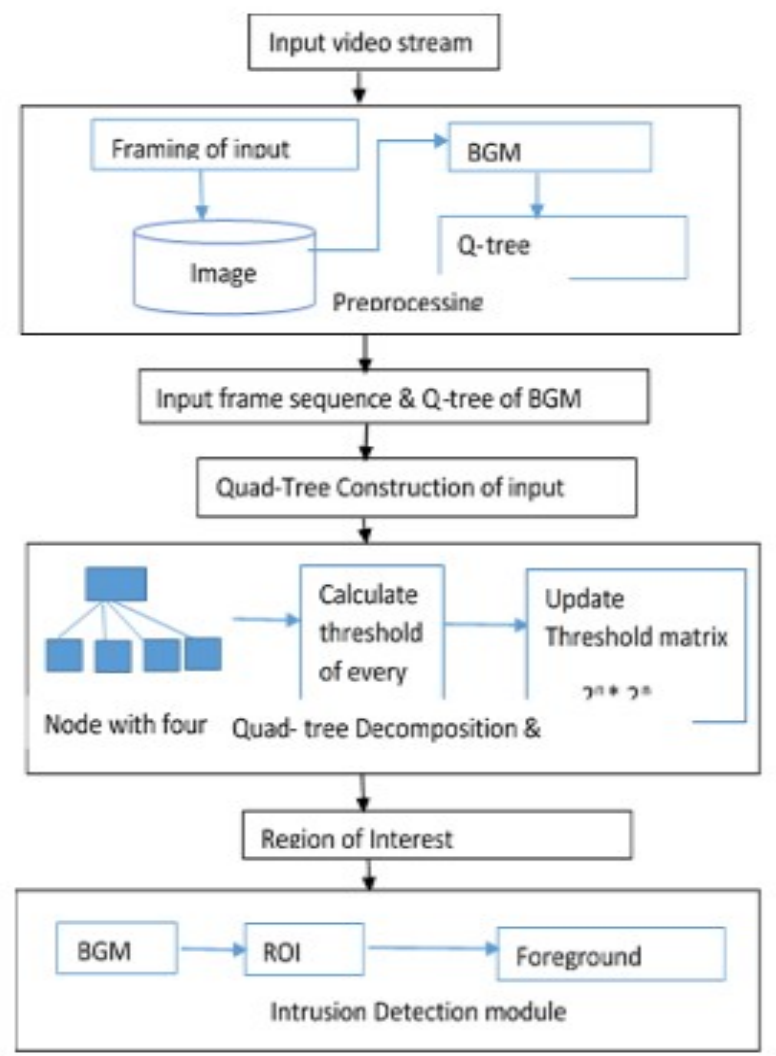

Fig. 3.2. General Architecture of the intrusion detection system.

of Interest based on the quadruple image segmentation for the input frame sequence (QTIS) and its matrix representation, fourth subsection depicts the classifier and background update policy.

The background modeling methods adopted in the literature $[1,2,9,33]$ etc. uses various parameter based as well non-parameter based background modeling which does its operation to every pixel in the frame. In this paper, we present a different technique through quadruple based image segmentation from where the foreground segmentation is done only to the specific Q-Tree node in the background frame.

The given video stream is considered as the input and it is divided into frames and the defined function Init_BGM () initializes the background model based on the average of first $n$ input frames. It is followed by Q-Tree representation of the defined background model. Then for the input frames the Q-Tree representation is done as well as the matrix child and threshold of size $2 n \times 2 n$ will also get updated. Fig. 3.2 Depicts the flow involved in Adaptive intrusion detection system.

3.1. Preprocessing. The first step in the Adaptive intrusion detection system is the preprocessing which includes framing, background initialization, and Q-tree representation of the background model 


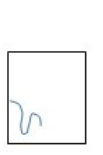

(a)

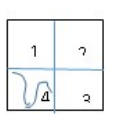

(b)

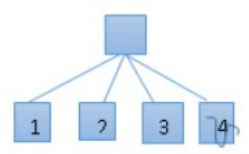

(c)

FIG. 3.3. (a) The background frame; (b) Q-Tree based image segmentation; (c) Tree representation segmented image.

\section{Framing}

The video stream is considered as the input and is divided into the frames suitable for further processing and is stored in the local database for the foreground identification and Intrusion detection.

2. Background Initialization

It is the first step but considered to be an important step in the process of background subtraction. There are various well know methods available to model the initial background. Few such is assigning the average illumination of first $n$ frames, or assigning the very first frame directly as BGM, or using the image order to approximate the background model, and the frame average over the time. Although the average of background frame that updates at an instance of time $t_{n}$ is familiar with many systems, it is not good enough to handle shadow and ghost problems. Here, we adopt a modest and proficient way of initializing the background where the median of first $n$ frames are considered. When considering the median of first $n$ frames, It also reflects the illumination change. At the initial time $t_{0}$, we set the initial background as the median of first $\mathrm{n}$ frames.

$$
\text { Init_BGM }=\operatorname{Median}\left(f_{k}\right)
$$

where $k$ varies from $1,2, \ldots, n$ at the timing instance $t_{0}$. The initial background plays a vital role in the process since the foreground detection that happens at timing instance $t_{j}$ depends on the background frame detected at $t_{0}$, where $j$ is closer to $n$.

3. Q-Tree representation of the Background model

The initial frame which is set as the background model is taken and is given with the Q-Tree representation. The background image is divided into regions with varying information. This is accomplished by the Q-Tree decomposition technique. Such decomposition is powerful as it gives homogeneous regions which in further gets constructed as a tree with each parent having maximum of four children. Each child corresponds to a quadrant of the segmented image frame as shown in Fig. 3.3.

The tree representation is also given with a coding procedure where 1 is given for the node with a child i.e. if node is a parent and 0 for child node which do not further have a child. So for the above tree in Fig. 3.3(c), the Q-Tree code(QTC) is given as 1-0000.

3.2. Q-Tree Decomposition and its Representation. Assuming the size of image array is $m \times n$, the entire image array is represented by quadruple tree. The Q-tree will have four child representing the four quadrants of the array and each of the child will be subdivided in a recursive manner. The procedure terminates the Q-Tree with leaves with homogeneous regions or until the specified level. These leaves correspond to the unique regions of the image segments.

Here the root of the tree corresponds to the entire image. When Q-tree iteration takes place at the first iteration, the image is segmented into four quadrants. Thus, resulting the tree with four homogeneous leaves. The iterations can be further continued until desired level or desired pixels are segmented. A sustainable condition is set by finding the pixel intensity difference and must be lesser than the threshold. The difference between high intensity pixel and low intensity pixel is calculated within the node of the decomposed quad tree and is ensured against the sustainability condition. Each level in the decomposed tree is given with $2 n \times 2 n$ nodes for the given integer $\mathrm{n}$, where $\mathrm{n}$ corresponds to the number of iterations. For example, at 0 split the whole image is represented as the singe node. At 1st split the image is segmented into $21 \times 21$ nodes which represents the four quadrants of the parent image frame.

Each node has left and right segments denoted by $l, r$, and top, bottom segments denoted by $t, b$, respectively. So each image is denoted by four disjoint set as nodes and are represented by $t l, t r, b l, b r$ in the clockwise order. 
Also for $n$ splits, the total number of nodes in the tree is given by formula,

$$
\text { No. of nodes in the tree }=\frac{4^{n}-1}{3}
$$

Since the scope of the research work is restricted to intrusion detection, we limit the value of $\mathrm{n}$ to 2 .So that maximum of two levels are possible and the level 0,1,2 corresponds to whole image, image with 4 quadrants and image with 16 regions respectively.

Q-Tree decomposition is performed by considering the input image frame of size $\mathrm{m} \times \mathrm{n}$. At each iteration, it divides the image frame into four quadrants and assign it as four children to the parent node. The same gets iterated for every child node until desired pixels are selected. At each iteration it updates the array $Q T C$ and QTT which corresponds to Q-Tree code and Q-Tree node threshold, respectively.

$$
Q T C_{i}\left[2^{m}\right]\left[2^{n}\right]=\left\{\begin{array}{ll}
1 & \text { for parent node } \\
0 & \text { for child node }
\end{array} \text { for all } 0 \leq m \leq 2,0 \leq n \leq 2 .\right.
$$

$$
Q T T_{i}\left[2^{m}\right]\left[2^{n}\right]=\{\text { adaptive threshold of the specific node }\} \text {, for } 1 \leq m \leq 2 m, 1 \leq n \leq 2 n .
$$

In the above equation $Q T C_{i}$ is the array which holds vale 1 for parent node and 0 for child node. Thus $Q T C_{0}$ holds 0 since there is no splitting, $Q T C_{1}$ holds 4 values in a two dimensional array [21], $Q T C_{2}$ holds 16 values as it corresponds to [22], $4 \times 4$ matrix array. Similarly $Q T T_{i}$ corresponds to the respective adaptive threshold of the node. $Q T C_{i}$ has been given with a third value 2 when every pixel in the region is a foreground pixel. The algorithm for Q-Tree decomposition is as follows.

Algorithm: Q-Tree Decomposition

Input: Input Frames

Output: Q-Tree segmented image frame with $Q T C$ and $Q T T$

Step 1: Ensure $I$ is the Image frame

Step 2: Check whether the dimension of $I$ is $m \times n$

Step 3: Perform the Quad tree decomposition of $I$

Step 4: Update the array $Q T C$ and $Q T T$, for every iteration $i$ according eqs (3.1) and (3.2)

Step 5: Calculate the pixel intensity $P_{i}$ as follows

$$
P_{i}(x, y)=\frac{1}{4} \sum_{m=0}^{1} \sum_{n=0}^{1} x_{i}-1(2 x+m, 2 y+n)
$$

Step 6: for $m, n=0,1,2$ if $P_{i-1}(2 x+m, 2 y+n)$ are leaves go to step 5

Step 7: Check equation in 5-6 for sustainability condition

$\left|P_{i}(x, y)-P_{i-1}(2 x+m, 2 y+n)\right|<T$

If true, set as leaf

Otherwise set as node

Step 8:continue step 5 for next x,y

End procedure

3.3. Finding ROI In Background Model. Most of the state of art background modeling methods keep only single background frame for foreground segmentation, in which the result of foreground detection consumes the whole frame. This procedure is termed as FBM ( frame-based background modeling). The inadequacy of the background modeling process opens a large room for the scholars to post their ideas. Many times the background modelling faces a problem where the part of the generated background may be inaccurate because of the problems like slow moving objects, image ghost and the shadows. The next problem is that the accuracy of background update may get worse as the frame distance increases, most cases this varies with frame compression rate. To solve these complications, we come out with a region- based background model (RBM). The enhancements that are noticed with the proposed method are at spatial level where foreground generation and segmentation mechanism performed on the basis of ROI but not entire frame. The other improvement is, in temporal level, where background generation and modeling are performed for every frame but not a whole video sequence.

Some of the literature discussed are the block based methods like Heikkila et.al.[71] proposed a method that models the scattering of block texture in a frame using the local binary pixel outline method. Moreover, in 
these block-based methods [71, 72, 73] there is a recurring problem of dealing with the entire frame. Although they consider the frame as a gathering of blocks, they perform their operation to entire block segments and reassemble those blocks back to frames.

To avoid these problems, we offered an algorithm to find the region of interest in the background to which the foreground segmentation is constrained. This approach presents two main ideas where it works on a Q-Tree of the background model. $Q T C$ is the code given for the regions in the image segments. For the point $P$ on the frame $f$, the coordinates are given as $P_{i, j}$ and the point $P$ on the background is given as $B_{i, j}$ and the pixel intensity difference is given as $D_{i, j}$. When there is the change in intensity i.e. $\left|D_{i, j}\right| \neq 0$, then it is classified as foreground pixel. On the other hand, If there is no change in the intensity where $\left|D_{i, j}\right|=0$, then $Q T C$ code is set as follows.

Procedure: Find_ROI()

Input : Q-tree of frame $f$ in the video stream

Output : Segmented foreground.

Algorithm:

Step 1: Read $Q T C_{i}\left[2^{m}\right]\left[2^{n}\right], Q T T_{i}\left[2^{m}\right]\left[2^{n}\right]$;

Step 2: $\quad$ check for $Q T C_{i}\left[2^{m}\right]\left[2^{n}\right]$; for $i=0,1,2$;

Step 3: If $\operatorname{code}=0$, the region has no intrusion;

Not a ROI

Classify every pixel in the region as background pixel;

Step 4: If code=1, the region has some intrusion;

Is a ROI

Collect coordinates within the region and calculate change in Intensity

$D_{i, j}=\left|P_{i, j}-B_{i, j}\right|$

if $\left|D_{i, j}\right| \neq 0$ then

classify as foreground,

else

end if

classify as background;

Step 5: Goto step 2 for the next $i$;

End procedure

The above procedure is used to check for the intensity change which corresponds to the foreground appearance, illumination change and many other issues.

3.4. Foreground Segmentation And Background Update. Once the ROI is determined, the next step is to perform the foreground segmentation. The colossal of the existing method includes ViBe, PBAS, Word consensus model. The ViBe set the first frame as background and foreground segmentation is done using the fixed threshold. This restriction holds back the adaptability of the method. PBAS, the non-parametric background model integrates the ideas of state-of-art foreground detection methods and works in a controlled environment. Here we follow the basic idea of ViBe, PBAS with some variations to provide an efficient segmentation. Fixed threshold of ViBe is replaced by an adaptive threshold, and the first frame as background model is over laced by the median of first $\mathrm{n}$ frames. To update the background model, we use the adjustable learning rate concept from the PBAS with a random scheme and dynamic threshold is used to identify the foreground. The algorithm has two important factors: $T(x), L(x)$ which corresponds to the dynamic threshold and learning rate for background update The background model $B_{i}(x)$ for the pixel $x$ is defined as $B(x)$.

$$
B(x)=\{B 1(x), \cdots, B k(x), \cdots B n(x)\}, \text { where } 1<k<n
$$

Pixel $x$ is considered to be the foreground pixel when the given condition holds true

$$
f(x)=\left\{\begin{array}{cc}
1 & \text { if } \operatorname{dist}((I x),(B x))<T(x) \\
0 & \text { otherwise }
\end{array}\right.
$$


The above formula illustrates that $f(x)$ is set to 1 , i.e. the pixel is classified as foreground, when the distance between the current frame and the background frame is lesser than the dynamic threshold. Otherwise, set with 0 means the pixel $x$ is the background pixel.

The dynamic threshold changes its value at each frame over the time instance tn, for nth frame. It is calculated by the formula as suggested in the literature [74].

$$
\Delta T=\alpha \frac{1}{w \cdot h} \sum_{i=0}^{n-1}|I(x)-B(x)|
$$

Since it changes with time, it is considered to be dynamic and the pixel we use the notation delta $T, \alpha$ is the coefficient called inhibitory coefficient, whose reference value is $2 . w \cdot h$ represents the width and height of the frame which in turn represents the size of the frame. The algorithmic representation is given below.

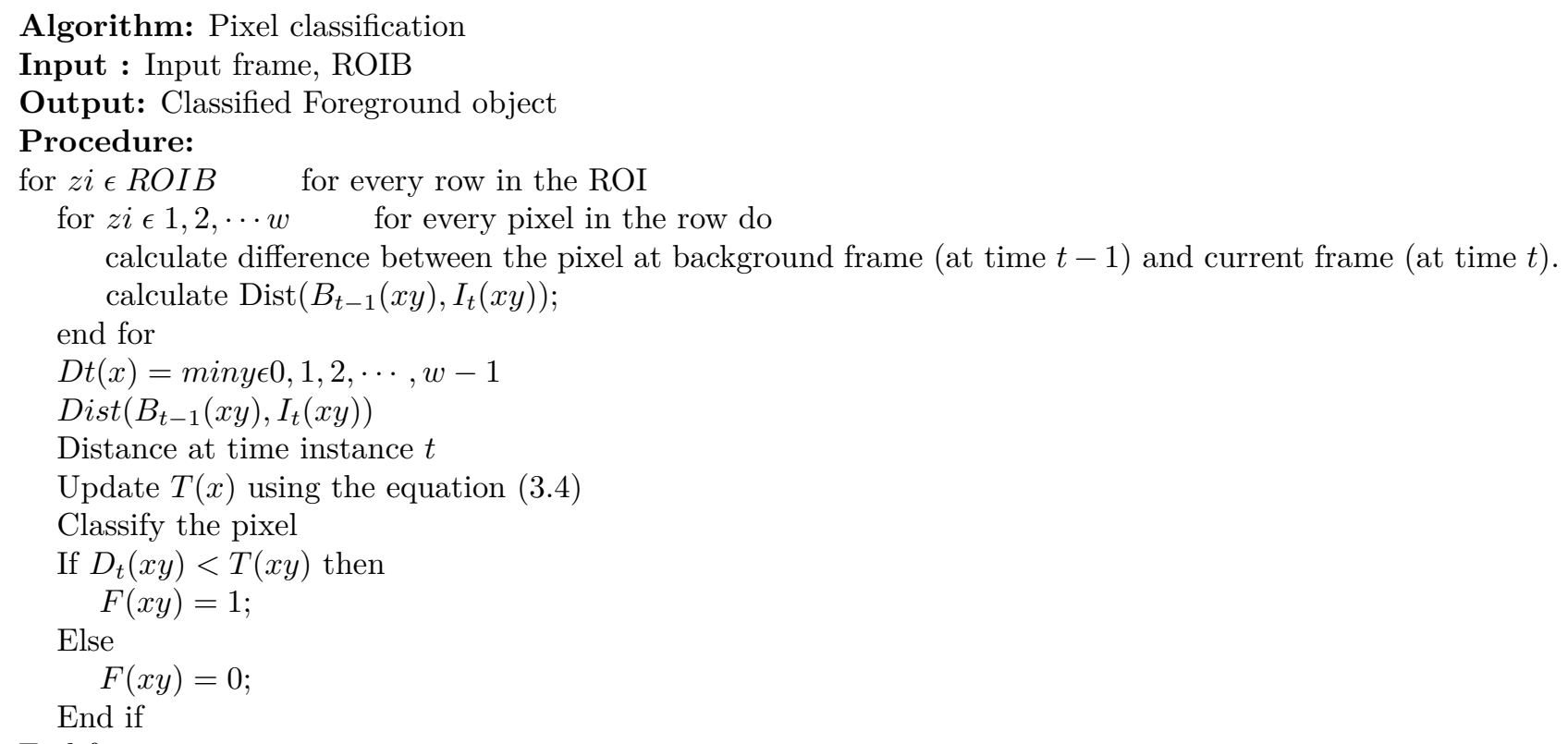

End for

End procedure.

The above procedure is used to perform the background subtraction only to the region of interest predicted in the previous step. The procedure is repeated for every line in the ROI and for every pixel x of the line the distance is calculated, then compared with dynamic threshold, $Q T T_{i}\left[2^{m}\right]\left[2^{n}\right]$. If the intensity difference is lesser than the threshold then it is classified as foreground otherwise classified as background.

As proposed in $\mathrm{ViBe}$, the update mechanism used is the conservative type where it never includes the pixel to the background which belongs to the foreground. Thereby, avoids the misclassification and keeps the false positive minimum.

4. Experiemental Analysis. Hereby, we discuss the quantitative and qualitative performance evaluation of the proposed technique. For which we presented the foreground segmentated image as qualitative evaluation. Then, parameter based metrics evaluation compared with the results of five other recent state-of-art techniques.

4.1. Evaluation metrics and the Test Dataset. The video sequences of the Change Detection Challenge 2014 dataset [75] has been used to assess the execution performance of the proposed approach both as a qualitative and quantitative evaluation. Since it is confirmed in many of the works, it is clear that traditional datasets are cramped and lacks challenging video streams. Moreover, standard methods have outperformed and no longer face the challenges of current video surveillances application.

The Change Detection Challenge 2014 dataset has been promised with 53 videos of 11 categories including the states of both outdoor as well indoor videos. These videos have been prearranged under categories Base- 
line, dynamic background, camera jitter, Intermittent object motion, shadows, thermal signatures, challenging weather, low frame-rate, night videos, PTZ capture and air turbulence. The baseline is considered to be an important category that includes the following challenges: background motion, isolated shadows, having uncontrolled objects and ghost. The intermittent object motion is the category that has the video with moving object stays static for some time and again started to move. To measure the accuracy, we calculated few metrics namely F-measure, percentage of correctness.

Classification (PCC) and Matthew's correlation coefficient (MCC) metrics are referred in the literatures F-Measure[SOIR], PCC[word consensus model], MCC and are calculated using terms true positive (TP), true negative (TN), false positive (FP), false negative (FN). To calculate the metrics it is assumed like foreground as positive and background as negative. When it is correctly detected then true, otherwise it is false. Thus, True positive is the number of pixels where foreground pixels are correctly detected as the foreground, false positive depicts the number of background pixels wrongly detected as the foreground, True negative represents background pixels correctly detected as the background and False negative represents the number of foreground pixels wrongly detected as background. These metrics in turn are used to calculate precision and recall.

Precision is the foreground prediction which is measured as the ratio of correctly classified foreground pixels to the total number of pixels classified as foreground pixels which include the misclassification.

$$
\text { Precision }=\frac{T P}{T P+F P}
$$

The precision is calculated since it gives the ability of the method to identify foreground pixels (considering misclassified positive class). The Recall measures the detection rate and is calculated as the ratio of correctly classified foreground pixels to the total number of foreground pixels in the ground truth (i.e. ratio of true positives to actual foreground pixels in the test image).

$$
\text { Recall }=\frac{T P}{T P+F N}
$$

The above two metrics namely precision and Recall are used in calculating the evaluation metrics like F-measure, PCC, MCC which are used to assess the quality of a method; As just, the method is considered if it has high Recall values, without losing Precision. F-Measure is the metric obtained as the weighted harmonic average of the values recall and precision. It is the metric used in most of the datasets to rank the methods. It is defined as follows:

$$
F_{\text {Measure }}=2 \cdot \frac{\text { Recall } \cdot \text { Precision }}{\text { Recall }+ \text { Precision }}
$$

The metric percentage of correct classification (PCC) is also used to determine whether both the background and foreground is classified correctly. For an effective foreground segmentation, PCC value must hold high. The PCC value is calculated using the below given equation:

$$
P C C=\frac{T P+T N}{T P+T N+F P+F N}
$$

The last metric used for evaluating the performance is Matthew's Correlation Coefficient(MCC) scores. As stated in the literature [2], it is found to assess the overall performance of unbalanced binary classification, into which the background subtraction comes in. it is calculated using the formula

$$
M C C=\frac{(T P * T N)-(F P * F N)}{\sqrt{(T P+F P) *(T P+F N) *(T N+F P) *(T N+F N)}}
$$

4.2. Results of challenge change detection 2014 dataset.. We compared our method to six other recent foreground segmentation methods, the adaptive background modeling(ABMFS)[78], the word consensus model(PAWCS) [77], the Gaussian mixture model (GMM) [6], the sample consensus background model (SACON) [17], ViBe [76], the pixel-based adaptive segmentation (PBAS) [79], the boosted Gaussian model 


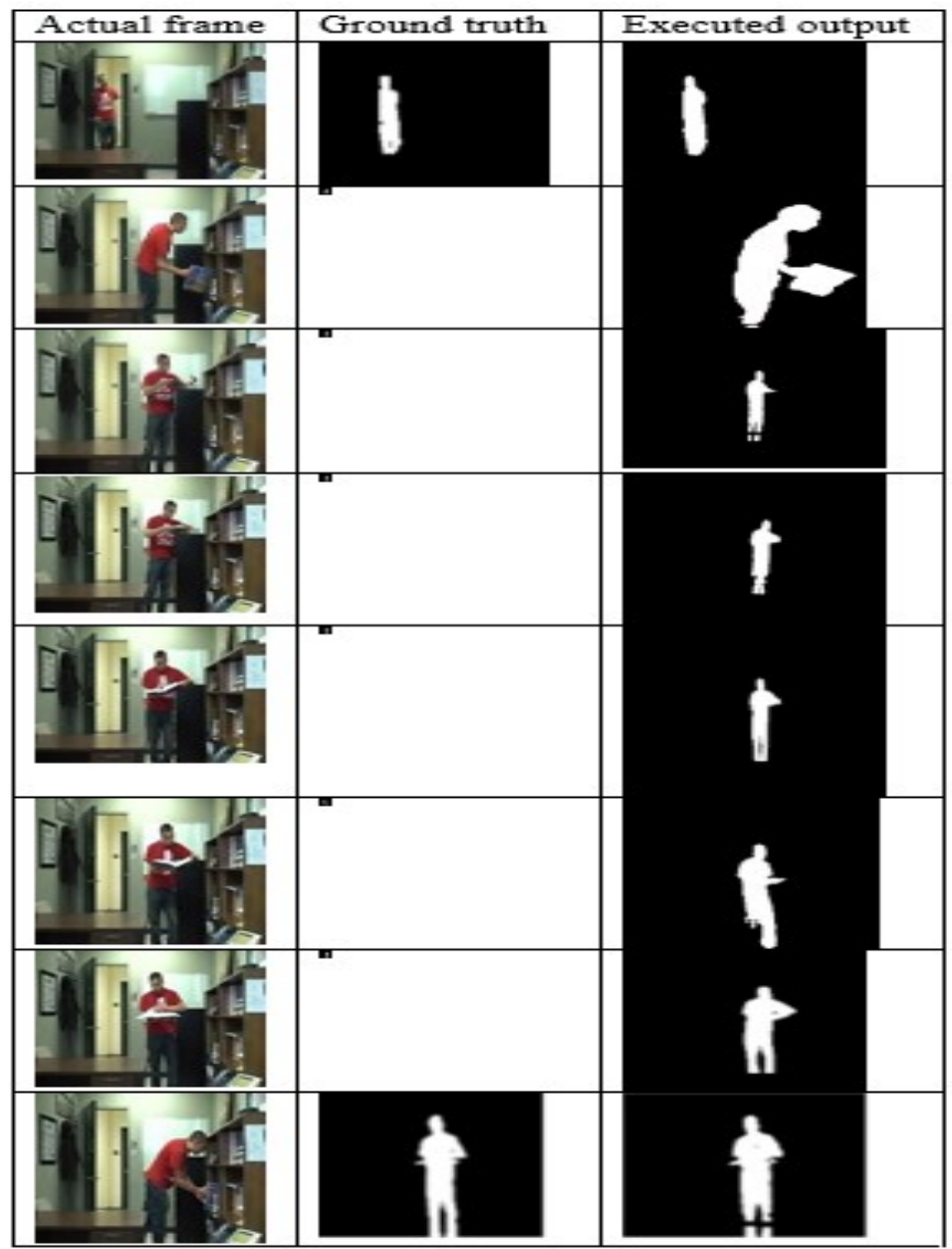

Fig. 4.1. General Architecture of the intrusion detection system.

(BMOG), and multimode background subtraction method(UMBS). Gaussian Mixture Model is a pixel-based parametric method and BMOG are pixel and blob-based parametric method. ABMFS and PAWCS are pixelbased nonparametric techniques, and are the two top performing foreground detection methods reported [20]. We integrate the region based background subtraction to the basics of pixel based adaptive segmentation method (PBAS).

For the execution result, the code available in OpenCV Library for GMM is used [6]. The codes for PBAS, ABMFS, PAWCS were provided by the authors and the parameters are also set as suggested by the authors respectively. The output of BMOG,GMM, UMBS and SubSENSE are presented as posted in the CDnet2014 dataset by the authors. Some of the reference values are taken directly from the papers.

Here we have taken only the video categories of baseline, Intermittent object and shadow. Since our work is restricted to the Closed room Intrusion Detection Technique we consider only indoor videos from the dataset and qualitative cum quantitative comparisons are done, proposed method obtained agreeable results for the different videos including shadow video. The other foreground detection methods used for comparison are all executed for the frame level operations Wherein the proposed method is operated only in the region of interest focused by Q-Tree code and seems to have acceptable foreground segmentation neared to other state of art methods. However, most segmentation methods classify some of the background pixels as foreground. The proposed method possibly obtains nearly correct foreground objects. 
TABLE 4.1

Average quantitative values of evaluation metrics from change detection dataset

\begin{tabular}{|l|c|l|l|l|}
\hline Scenarios & $\begin{array}{c}\text { Percentage of } \\
\text { correctness } \\
\text { classification }\end{array}$ & Recall & Precision & F-measure \\
\hline Bad Weather & 98.5 & 0.604 & 0.315 & 0.411 \\
\hline Baseline & 96.0 & 0.901 & 0.849 & 0.874 \\
\hline Camera Jitter & 93.8 & 0.791 & 0.359 & 0.495 \\
\hline Dynamic Background & 99.1 & 0.655 & 0.200 & 0.317 \\
\hline Intermittent motion object & 97.5 & 0.815 & 0.835 & 0.824 \\
\hline Low frame & 89.7 & 0.640 & 0.645 & 0.642 \\
\hline Night Video & 92.0 & 0.802 & 0.502 & 0.616 \\
\hline PTZ & 84.5 & 0.725 & 0.287 & 0.410 \\
\hline Shadow & 96.8 & 0.914 & 0.503 & 0.649 \\
\hline Thermal & 98.5 & 0.865 & 0.623 & 0.727 \\
\hline Turbulence & 97.4 & 0.710 & 0.559 & 0.625 \\
\hline Overall & 94.9 & 0.763 & 0.516 & 0.615 \\
\hline
\end{tabular}

Another quantitative comparative approach on experimental result on the Change Detection Challenge dataset is presented were we broadly test the proposed method. Here, the scenario considered includes Baseline, Intermittent motion objects, and Shadows. The other category usage is suppressed due to the scope of the research.

The qualitative analysis is given above with representation of actual frame, its ground truth and the executed output. Table 4.1 depicts the average values of the metrics Recall, Precision, F-measure and percentage of correctness classification. It projects the average value from the CDnet14 dataset for all the categories of videos in the open dataset. These average metric values are used for comparison of the proposed algorithm. Though at some places the proposed algorithm can not achieve remarkable enhancement, it goes above the average from the dataset.

Table 4.2 presents evaluation metrics F-Measure score of different video criteria considered for our method in comparison with six other high performing methods on the CDnet 2014 dataset. The values of top three performing methods are given in red, blue and yellow color shade. Though our method performed best only for the videos pedestrian and copy machine, it is clearly visible that the performance of other criteria office, parking, bus station is also evaluated to very near of well performed methods The proposed modeling method could acclimate to speedy changes caused by slow background changes, as well to illumination variation and static objects since it can execute more number of frames per second. Though, the performance is not good for the category sofa, the overall scores proven to be the best performance. For the same the graphical representation is given in Fig.4.

Table 4.3 also shows in detail the PCC metric values in the compared state of specific criteria Baseline, Intermittent motion object and Shadows respectively. It could be proven that our method obtained higher scores in every criteria at least for one video frame sequences. The top three values are represented with bold red, blue and yellow respectively. Table 4.4. Also represent the MCC( Mathew's Correlation Coefficient) with its graphical representation in Fig.4.3

5. Conclusion and Future Work. In this paper, we proposed an adaptive and efficient background modeling technique that exploits region based background subtraction. It also provides quick response to the real time scenario with data inconsistency. Furthermore, we have introduced "Q-Tree based foreground segmentation" algorithm to model the background that selects the specific region in a faster manner and promotes efficient foreground segmentation. This selection mechanism works well in most situations with varying conditions. The experimental result shows that the overall performance of proposed method is better than the entire benchmark systems considered. The method was proposed to identify the moving object in the region of interest, and to perform pixel based background subtraction for foreground detection. Satisfactorily, 
TABLE 4.2

Comparitive F-Measure values of the selected methods from CDnet14

\begin{tabular}{|l|l|l|l|l|l|l|}
\hline Method / Video & \multicolumn{2}{c|}{ Baseline } & Intermittent object & \multicolumn{2}{c|}{ Shadow } \\
\hline \multicolumn{1}{|c|}{ Category } & Pedestrian & Office & Parking & Sofa & $\begin{array}{c}\text { Copy } \\
\text { Machine }\end{array}$ & Bus Station \\
\hline proposed & 0.981 & 0.959 & 0.768 & 0.734 & 0.940 & 0.891 \\
\hline ABMFS & 0.957 & 0.972 & 0.618 & 0.846 & 0.871 & 0.869 \\
\hline PAWCS & 0.946 & 0.937 & 0.819 & 0.725 & 0.914 & 0.973 \\
\hline GMM & 0.960 & 0.656 & 0.482 & 0.652 & 0.660 & 0.787 \\
\hline BMOG & 0.920 & 0.626 & 0.698 & 0.632 & 0.640 & 0.823 \\
\hline SUBSENSE & 0.954 & 0.963 & 0.4447 & 0.742 & 0.929 & 0.864 \\
\hline UMMBS & 0.938 & 0.965 & 0.672 & 0.769 & 0.807 & 0.677 \\
\hline
\end{tabular}

TABLE 4.3

Comparative PCC values of the selected methods from CDnet144

\begin{tabular}{|l|l|l|l|l|l|l|}
\hline Method / Video & \multicolumn{2}{c|}{ Baseline } & Intermittent object & \multicolumn{2}{c|}{ Shadow } \\
\hline \multicolumn{1}{|c}{ Category } & Pedestrian & Office & Parking & Sofa & $\begin{array}{c}\text { Copy } \\
\text { Machine }\end{array}$ & Bus Station \\
\hline proposed & 99.962 & 99.433 & 97.932 & 97.934 & 99.160 & 99.022 \\
\hline ABMFS & 99.916 & 99.616 & 94.507 & 98.747 & 98.239 & 98.995 \\
\hline PAWCS & 99.892 & 99.166 & 97.323 & 98.075 & 98.869 & 99.051 \\
\hline GMM & 99.919 & 96.332 & 95.603 & 97.608 & 96.113 & 98.584 \\
\hline BMOG & 99.833 & 95.510 & 96.168 & 97.222 & 95.729 & 98.770 \\
\hline SUBSENSE & 99.912 & 99.491 & 94.456 & 98.115 & 99.047 & 98.945 \\
\hline UMMBS & 99.871 & 99.517 & 95.335 & 98.216 & 97.675 & 98.174 \\
\hline
\end{tabular}

proposed method performed the task of foreground segmentation in the identified region of interest with a reduced mis-classification which results in better scores for the metrics considered. Thus, the quantitative performance is achieved. However, method shows lack in performance for the criteria shadow elimination, Baseline of CDnet2014. We will attempt to solve these issues using adaptive threshold for specific region based on the learning rate to the specific region.

6. Acknowledgement. We thank the team who made the website (www.changedetection.net) available and for providing the resource to test and compare our method.

\section{REFERENCES}

[1] C. Wren, A. Azarhayejani, T. Darrell, A.P. Pentland, IEEE Trans. PatternAnal. Mach Intell. 19 (7) (1997) $780-785$.

[2] M. Piccardi, IEEE Int. Conf. Syst. Man Cybern 4 (Oct. 2004)3099-3104. The Hague, The Netherlands.

[3] Y. Benezeth, P.M. Jodoin, B. Emile, H. Laurent, C. Rosenberge, Review and evaluation of commonly-implemented background subtraction al- gorithms, in: Proc. IEEE Int. Conf. Pattern Recognit, Dec.2008, pp. 1-4.

[4] C. Stauffer, E. Grimson, Adaptive background mixture models for real-time tracking, in: Proc. IEEE Int. Conf. Comput. Vis. Pattern Recog- nit., vol. 2, 1999, pp. 246-252.

[5] C. Stauffer, E. Grimson, IEEE Trans. Pattern Anal. Mach. Intell. 22 (8)(Aug. 2000) $747-757$.

[6] Z. Zivkovic, Improved adaptive Gaussian mixture model for background subtraction, in: Proc. 17th Int. Conf. Pattern Recognit., vol. 2, Aug. 2004, pp. 28-31.

[7] D.S. LeE, Pattern Anal Mach. Intell. IEEE Trans. 27 (5) (May 2005)827-832.

[8] A. Shimada, D. Arita, R.I. Taniguchi, Dynamic control of adaptive mixture-of-Gaussians background model, in: AVSS '06 Proceedings ofthe IEEE International Conference on Video and Signal Based Surveil- lance, IEEE Computer Society Washington, DC, USA, 2006.

[9] A. Elgammal, D. Hanvood, L.S. Davis, Non-parametric model for background subtraction, in: Proc. ECCV 2000, June 2000, pp. $751 \mathrm{e} 767$.

[10] N.M. Oliver, B. Rosario, A.P. Pentland, IEEE Trans. Pattern Anal. Mach.Zntell. 22 (8) (2000) $831-843$. 
TABLE 4.4

Comparative MCC values of the selected methods from CDnet14

\begin{tabular}{|l|l|l|l|l|l|l|}
\hline Method / Video & \multicolumn{2}{c|}{ Baseline } & Intermittent object & \multicolumn{2}{c|}{ Shadow } \\
\hline \multicolumn{1}{|c|}{ Category } & Pedestrian & Office & Parking & Sofa & $\begin{array}{c}\text { Copy } \\
\text { Machine }\end{array}$ & Bus Station \\
\hline proposed & 0.981 & 0.956 & 0.857 & 0.747 & 0.936 & 0.887 \\
\hline ABMFS & 0.956 & 0.970 & 0.591 & 0.842 & 0.862 & 0.865 \\
\hline PAWCS & 0.946 & 0.934 & 0.806 & 0.740 & 0.910 & 0.868 \\
\hline GMM & 0.960 & 0.672 & 0.459 & 0.667 & 0.657 & 0.785 \\
\hline BMOG & 0.921 & 0.611 & 0.698 & 0.626 & 0.628 & 0.818 \\
\hline SUBSENSE & 0.954 & 0.960 & 0.516 & 0.7487 & 0.924 & 0.860 \\
\hline UMMBS & 0.938 & 0.962 & 0.650 & 0.767 & 0.805 & 0.704 \\
\hline
\end{tabular}

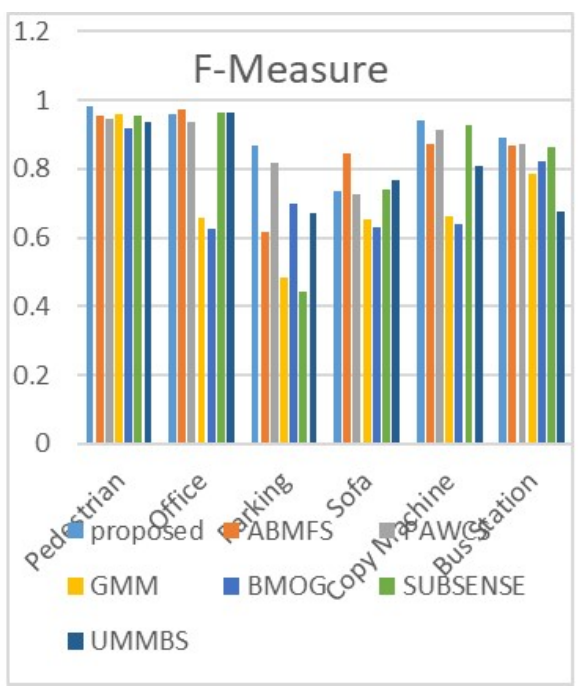

FIG. 4.2. Graphical representation of PCC

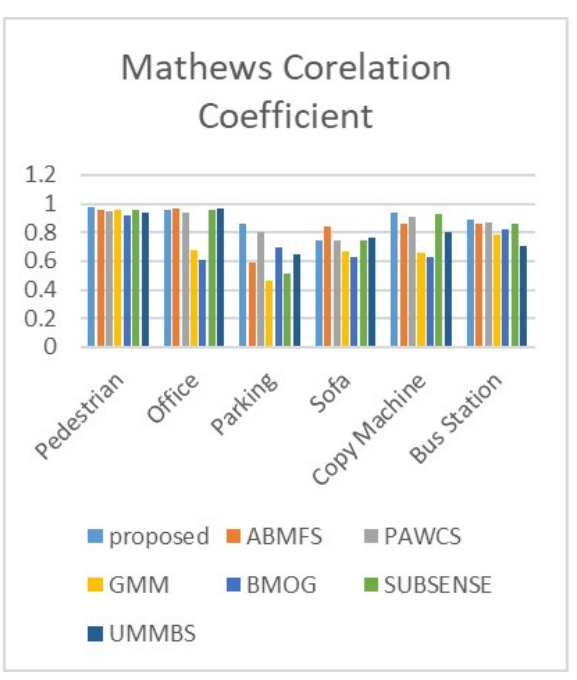

FIG. 4.3. Graphical representation of $M C C$. 
[11] O. Barnich, M. Van Droogenbroeck, Vibe: a powerful random technique to estimate the background in video sequences, in: IEEE Int. Conf. on Acoustics, Speech and Signal Processing, 2009, pp. 945e948.

[12] O. Barnich, M. Van Droogenbroeck, IEEE Transaction Image Process. 20 (6) (2011) 1709-1724.

[13] Y. Min-Hsiang, H. Chun-Rong, L. Wan-Chen, L. Shu-Zhe, C. Kun-Ta, IEEE Transaction Cirt.s Syst. Video Technol. 25 (4) (2015) 595-608.

[14] M. Van Droogenbroeck, O. Paquot, Background subtraction: experiments and improvements for ViBe, in: Proc. IEEE Comput. Soc. Conf. Comput. Vis. Ptrn Recognit. Workshops, Jun. 2012, pp. 32-37.

[15] M. Hofmann, P. Tiefenbacher, G. Rigoll, Background segmentation with feedback: the pixel-based adaptive segmenter, in: Proc. IEEE Comput. Soc. Conf. Comput. Vis. Pattern Recognit. Workshops, Jun. 2012, pp.38-43.

[16] K. Kim, T. Chalidabhongse, D. Harwood, L. Davis, Background modeling and subtraction by codebook construction, in: Int. Conf. on Image Processing, 2004, pp. 3061-3064.

[17] K. Kim, T. Chalidabhongse, D. Harwood, L. Davis, Real-Time Imaging 11 (3) (2005) 172-185.

[18] L. Maddalena, A. Petrosino, IEEE Transaction Image Process. 17 (7) (2008) 1168-1177.

[19] Y. Benezeth, P.M. Jodoin, B. Emile, H. Laurent, C. Rosenberge, J. Electron Imaging (July 23, 2010).

[20] A. McIvor, Background subtraction techniques, in: Proc. Image Vis .Comput., Auckland, New Zealand, Nov. 2000.

[21] R. Radke, S. Andra, O. Al-Kofahi, B. Roysam, IEEe Transaction on Image Process 14 (Mar. 2005) 294-307.

[22] S. Elhabian, K. El-Sayed, S. Ahmed, Recent Pattern. comput. Sci. 1 (Jan.2008) 32-54.

[23] T. Boummans, F. El Baf, B. Vachon, Statistical background modeling for foreground detection: a survey, in: Handbook of Pattern Recogn. and Computer Vision, vol. 4, World Scientific, Singapore, Jan. 2010, pp. 181-199 ch. 3.

[24] S. Panahi, S. Sheikhi, S. Hadadan, N. Gheissari, Evaluation of back- ground subtraction methods, in: Proc. Int. Conf. on Digital Image Computing: Techniques and Applications, IEEE, Piscataway, NJ, 2008, pp. 357-364.

[25] D. Parks, S. Fels, Evaluation of background subtraction algorithms with post-processing, in: Proc. of IEEE Int. Conf. on Advanced Video and Signal Based Surveillance, 2008, pp. 192-199.

[26] A. Sobral, BGSLibrary: an opencv cbp background subtraction library, in: IX Workshop de Viso Computacional (WVC'2013). Rio de Janeiro, Brazil, 2013. Software available at: http://code.google.com/p/bgslibrary/. [39] http://www.changedetection.net.

[27] A. Mittal, N. Paragios, Motion-based background subtraction using adap- tive kernel density estimation, in: Proc. Int. Conf. On Computer Vision and Pattern Recognition, IEEE, Piscataway, NJ, 2004, pp. 302-309.

[28] A. Elgammal, R. Duraiswami, D. Harwood, L. Davis, Proc. IEEE 90 (7) (Jul. 2002) 1151-1163.

[29] Y. Sheikh, M. Shah, IEEE Trans. Pattern Anal. Mach. Intell. 27 (11) (Nov. 2005) 1778-1792.

[30] Z. Zivkovic, F. van der Heijden, Pattern Recognit. Lett. 27 (May 2006)773-780.

[31] A. Tavakkoli, M. Nicolescu, G. Bebis, M. Nicolescu, Mach. Vis. Appl. 20 (Oct. 2008) 395-409.

[32] C. Ianasi, V. Gui, C. Toma, D. Pescaru, Facta Univ. Ser. Elec. Energ. 18 (1) (Apr. 2005) 127-144.

[33] A. Tavakkoli, M. Nicolescu, G. Bebis, An adaptive recursive learning technique for robust foreground object detection, in: ECCV 2006, May 2006.

[34] A. Tavakkoli, M. Nicolescu, G. Bebis, Robust recursive learning for foreground region detection in videos with quasistationary backgrounds, in: ICPR 2006, Aug. 2006.

[35] A. Tavakkoli, M. Nicolescu, G. Bebis, Automatic statistical object detection for visual surveillance, in: IEEE Southwest Symposium on Image Analysis and Interpretation, Denver, Colorado, SSIAI 2006, March 2006.

[36] T. Tanaka, A. Shimada, D. Arita, R. Taniguchi, Non-parametric back- ground and shadow modeling for object detection, in: ACCV 2007, Nov.2007, pp. 159-168.

[37] R. Ramezani, P. Angelov, X. Zhou, A fast approach to novelty detection in video streams using recursive density estimation, in: International IEEE Symposium on Intelligent Systems, vol. 2, Sept. 2008, pp. 142e147.

[38] T. Kohonen, Learning vector quantization, Neural Networks vol. 1, 1988, pp. 3-16.

[39] B.D. Ripley, Pattern Recognition and Neural Networks, CambridgeUniversity Press, 1996.

[40] A. Ilyas, M. Scuturici, S. Miguet, Real time foreground-background segmentation using a modified codebook model, in: Advanced Video and Signal Based Surveillance, 2009.

[41] Z. Zivkovic, F. van der Heijden, IEEE Trans. PAMI 26 (5) (2004). [55] Pattern Recognit. (2007) 1091-1105.

[42] H. Wang, D. Suter, Background Subtraction Based on a Robust Consensus Method, Proceedings of the 18th International Conference on Pattern Recognition (ICPR'06), pp. 223-226.

[43] L. Maddalena, A. Petrosino, The SOBS algorithm: what are the limits ?, in: CVPRW 2012, June 2012, pp. 21-26.

[44] K.E.A. Sande, T. Gevers, C.G.M. Snoek, IEEE Trans. Pattern Anal.Mach. Intell. 32 (9) (Sep. 2010) 1582-1596.

[45] P.-M. Jodoin, M. Mignotte, J. Konrad, IEEE Trans. Circuits Syst. Video Technol. 17 (12) (Dec. 2007) 1758-1763.

[46] T. Bouwmans, F. El Baf, B. Vachon, Recent Pat. Comput. Sci. 1 (3) (2008) 219-237.

[47] R. Cucchiara, M. Piccardi, A. Prati, IEEE Trans. Pattern Anal. Mach.Intell. 25 (10) (Oct. 2003) 1-6.

[48] E.L. Hall, Computer Image Processing and Recognition, Academic Press, 1979.

[49] S.-Y. Chien, W.-K. Chan, Y.-H. Tseng, H.-Y. Chen, IEEE Trans. Circuits Syst. Video Technol. 23 (6) (Jun. 2013) 921-934.

[50] M. Seki, T. Wada, H. Fujiwara, K. Sumi, Background subtraction based on cooccurrence of image variations, in: Proc. IEEE Comput. Soc. Conf. Comput. Vis. Pattern Recognit, Jun. 2003, pp. II-65eII-72.

[51] D. Russell, S. Gong, A highly efficient block-based dynamic background model, in: Proc. IEEE Conf. Adv. Video Signal Based Surveill, Sep.2005, pp. 417-422.

[52] H.-L. Eng, J. WANG, A.H.K.S. Wah, W.-Y. Yau, IEEE Trans. Image Process. 15 (6) (Jun. 2006) 1583-1600.

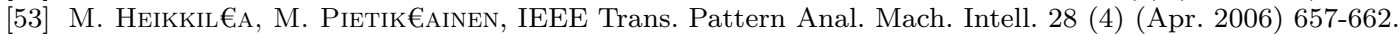

[54] W.-C. Liu, S.-Z. Lin, M.-H. Yang, C.-R. Huang, Real-time binary descriptor based background modeling, in: Proc. IAPR Asian Conf. Pattern Recognit, Nov. 2013, pp. 722-726. 
[55] G.-H. Huang, C.-R. Huang, Binary invariant cross color descriptor usinggalaxy sampling, in: Proc. 21st Int. Conf. Pattern Recognit, Nov. 2012, pp. 2610-2613.

[56] Y. Nonaka, A. Shimada, H. Nagahara, R. TAniguchi, Evaluation report of integrated background modeling based on spatio-temporal features, in: Proc. IEEE Comput. Soc. Conf. Comput. Vis. Pattern Recognit. Work- shops, Jun. 2012, pp. 9-14.

[57] S.-S. Huang, L.-C. Fu, P.-Y. Hsiao, IEEE Trans. Circuits Syst. VideoTechnol. 19 (4) (Apr. 2009) 522-532.

[58] T.H. Tsai, C.-Y. Lin, S.-Y. Li, IEEE Trans. Circuits Syst. Video Technol.23 (1) (Jan. 2013) 15-e29.

[59] A. Schick, M. Bauml, R. Stiefelhagen, Improving foreground segmen- tations with probabilistic superpixel Markov random fields, in: Proc. IEEE Comput. Soc. Conf. Comput. Vis. Pattern Recognit. Workshops, Jun. 2012, pp. 27-31.

[60] Y.-T. Chen, C.-S. Chen, C.-R. Huang, Y.-P. Hung, Pattern Recognit. 40 (10) (Oct. 2007) 2706-2715.

[61] M. Cristani, M. Bicego, V. Murino, Integrated region- and pixel-based approach to background modeling, in: Proc. Workshop Motion Video Comput, Dec. 2002, pp. 3-8.

[62] Y. Wang, P.-M. Jodoin, F. Porikli, J. Konrad, Y. Benezeth, P. Ishwar, Cdnet 2014: an expanded change detection benchmark dataset, in: Computer Vision and Pattern Recognition Workshops (CVPRW), 2014 IEEE Conference on. IEEE, 2014, pp. 393-400.

[63] T. Ojala, M. Pietikainen, T. Maenpaa, IEeE Trans. Pattern Anal. Mach.Intell. 24 (7) (Jul. 2002) 971-987.

[64] Z. Zivkovic AND F. van Der Heijden, "Efficient adaptive density estima tion per image pixel for the task of background subtraction," Pattern Recognit. Lett., vol. 27, no. 7, pp. 773-780, 2006.

[65] N. Goyette, P.-M. Jodoin, F. Porikli, J. Konrad, and P. Ishwar, "Changedetection.net: A new change detection benchmark dataset," in Proc. IEEE Comput. Soc. Conf. CVPRW, Jun. 2012, pp. 1-8.

[66] P. Kaew, Tra Kul Pong and R. Bowden, "An improved adaptive background mixture model for real-time tracking with shadow detection," in Video- Based Surveillance Systems. New York, NY, USA: Springer, 2002, pp. 135-144.

[67] J. Guo, C. Hsia, Y. Liu, M. Shin, C. Chang, And J. Wu, "Fast background subtraction based on a multilayer codebook model for moving object detection," IEEE Trans. Circuits Syst. Video Technol., vol. 23, no. 10, pp. 1809-1821, Oct. 2013.

[68] T. S. F. Haines And T. XIAng, "Background subtraction with Dirichlet process mixture models," IEEE Trans. Pattern Anal. Mach. Intell., vol. 36, no. 4, pp. 670-683, Apr. 2014.

[69] K. Kim, T. H. Chalidabhongse, D. Harwood, and L. Davis, "Real-time foreground-background segmentation using codebook model," Real- Time Imag., vol. 11, no. 3, pp. 172-185, Jun. 2005.

[70] Eli Shusterman,"Image compression via improved quadtree decomposition algorithm",IEEE Trans. On Image Processing,Vol.3,No.2,March 1994.

[71] Heikkila, M., and Pietikainen, M., 'A texture-based method for modelling the background and detecting moving objects', IEEE Trans.Pattern Anal. Mach. Intell., 2006, 28, pp. 657-662.

[72] Tian, G., AND Men, A., 'An improved texture-based method for background subtraction using local binary patterns'. Image and Signal Processing, (CISP), Tiajin, China, 2009.

[73] Liao, S., Zhao, G., Kellokumpu, V., Pietikainen, M., and Li, S., 'Modelling pixel process with scale invariant local patterns for background subtraction in complex scenes'. IEEE Conf. on Computer Vision and Pattern Recognition, (CVPR), San Francisco, CA, USA, 2010

[74] R.S.Rakibe, Prof.B.D.Patil, "Human motion detection using background subtraction algorithm," Int. Journal of Advance research in CS and SE., vol. 4, no. 2, pp. 45-48, Feb. 2014.

[75] N. Goyette, P. M. Jodoin, F. Porikli, J. Konrad, and P. Ishwar, "Changede- tection.net: A new change detection benchmark dataset," in Proc. IEEE Conf. Comput. Vis. Pattern Recognit. Workshops, Jun. 2012, pp. 1-8.

[76] O. Barnich and M. Van Droogenbroeck, "ViBe: A universal background subtraction algorithm for video sequences," IEEE Trans. Image Process., vol. 20, no. 6, pp. 1709-1724, Jun. 2011.

[77] Pierre-Luc St-Charles, Guillaume-Alexandre Bilodeau, and Robert Bergevin, "Universal Background Subtraction Using Word Consensus Models," IEEE Trans.on image processing, vol. 25, no.10, pp. 747-757, Oct. 2016.

[78] Zuofeng Zhong, Bob Zhang, Guangming Lu, Yong Zhao, and Yong Xu, "An Adaptive background modeling method for foreground segmentation," IEEE Trans. Intell. Transp. Syst., vol. 18, no. 5, pp. 1109-1120, May 2017.

[79] A. Sobral and A. Vacavant, "A comprehensive review of background subtraction algorithms evaluated with synthetic and real videos," Comput. Vis. Image Understand., vol. 14, pp. 4-21, May 2014.

[80] Ma, M., Hu, R., Chen, S., XiaO, J., \& Wang, Z.," Robust background subtraction method via low-rank and structured sparse decomposition". China Communications, 15(7), 156-167, July 2018.

[81] Roy, K., Arefin, M. R., Makhmudkhujaev, F., Chae, O., \& Kim, J. " Background Subtraction Using Dominant Directional Pattern". IEEE Access, 6, 39917-39926, December 2018.

[82] Mingliang Chen,Zing wei,Qingxiong Yang,Qing Li,Gang Wang, Ming-Hsuan yang, "Spatiotemporal GMM for Background Subtraction with superpixel Hierarchy," IEEE Trans. Pattern Anal. Mach. Intell., vol. 40, no.6, pp. 1518- 1526, June 2018.

Edited by: Swaminathan JN

Received: Sep 12, 2019

Accepted: Dec 9, 2019 